\title{
NOTES ON DIFFERENTIAL CALCULUS IN TOPOLOGICAL LINEAR SPACES, III
}

\author{
S. YAMAMURO \\ (Received 1 July 1974) \\ Communicated by E. Strzelecki
}

Throughout this note, let $E, F$ and $G$ be locally convex Hausdorff spaces over the real number field $R$. We denote real numbers by Greek letters. The sets of all continuous semi-norms on $E$ and $F$ will be denoted by $P(E)$ and $P(F)$ respectively, and $A$ will always stand for an open subset of $E$.

The purpose of this note is to investigate a problem about the differentiability proposed by Hyers (1945). The problem is the differentiability of the inverse map of a differentiable map under natural conditions. In the previous notes (Yamamuro, to appear; 1975) we have considered the same problem for different differentiabilities-the Fréchet and strong Fréchet differentiability. For the definitions and fundamental properties of these two differentiabilities, we refer to Yamamuro (1974). The Hyers differentiability is stronger than Fréchet differentiability and weaker than strong Fréchet differentiability, and as we shall show in this note, it does not behave well when the differentiability of the inverse map is involved.

\section{Definition of Hyers differentiability}

A map $f: A \rightarrow F$ is said to be Hyers differentiable at $a \in A$ if there exists a continuous linear map $u$ of $E$ into $F$ such that, for

$$
r(f, a, x)=f(a+x)-f(a)-u(x),
$$

the following condition is satisfied:

for any $q \in P(F)$ there exists $p \in P(E)$ such that

$$
\lim _{\varepsilon \rightarrow 0} \sup _{p(x) \leq 1} q\left(\varepsilon^{-1} r(f, a, \varepsilon x)\right)=0 .
$$


If this is the case, the map $u$ is determined uniquely; we call it the Hyers derivative of $f$ at $a$ and denote it by $f^{\prime}(a)$. Since the semi-norm $p$ in the above condition can be replaced by any continuous semi-norm which is larger than $p$, we can always suppose that $a+\bar{U}_{p} \subset A$, where $\bar{U}_{p}=\{x \in E ; p(x) \leqq 1\}$. When $E$ and $F$ are normed spaces, this coincides with the usual Fréchet differentiability.

(1.1) The above condition is equivalent to the following: for any $q \in P(F)$ there exists $p \in P(E)$ such that (1) $p(x)=0$ implies $q(r(f, a, x))=0$ and (2) $p(x)^{-1} q(r(f, a, x)) \rightarrow 0$ when $p(x) \rightarrow 0$ with $p(x) \neq 0$.

It immediately follows from this that the map $f$ is continuous at $a$. However, the continuity in the usual sense is not suitable to this differentiability. One evidence for this is the following fact. Let $f: A \rightarrow F$ be Gateaux differentiable at every point of $A$ and denote the derivatives by $f^{\prime}(x)$. Then, $f^{\prime}$ maps $A$ into $L(E, F)$, the set of all continuous linear maps of $E$ into $F$. We denote by $L_{b}(E, F)$ the space $L(E, F)$ equipped with the topology of uniform convergence on bounded sets. If $f^{\prime}: A \rightarrow L_{b}(E, F)$ is continuous at $a$, then $f$ is Fréchet differentiable at $a$, but, as we have shown in Yamamuro (1974; page 52), it is not necessarily Hyers differentiable there. It appears that each differentiation has its own continuity that is most naturally connected to its definition.

Temporarily, we shall say that $f: A \rightarrow F$ is $(H)$-continuous at $a \in A$ if for any $q \in P(F)$ there exists $p \in P(E)$ such that $q\left(f\left(a+x_{\wedge}\right)-f(a)\right) \rightarrow 0$ whenever a net $\left(x_{\lambda}\right)$ satisfies $p\left(x_{\lambda}\right) \rightarrow 0$. It is obvious that $(\mathrm{H})$-continuity implies continuity and, moreover, if $f^{\prime}$ is (H)-continuous at $a$ in the above question, then $f$ is Hyers differentiable at $a$.

The following fact follows immediately from (1.1).

(1.2) If $f: A \rightarrow F$ is Hyers differentiable at $a \in A$, then $f$ is $(H)$-continuous at $a$.

Now, any differentiability which implies continuity does not always satisfy the chain rules of more than second order, because the composition map $(u, v) \rightarrow v \circ u$ of $L_{b}(E, F) \times L_{b}(F, G)$ into $L_{b}(E, G)$ is not continuous unless $F$ is normable. In other words, it is impossible for such differentiability to consider a category whose morphisms are more than twice differentiable. However, the following fact has been observed by, for instance, Keller (1963-64).

(1.3) The Hyers differentiability satisfies the first order chain rule.

\section{Maps of type $\left(D^{-1}\right)$ in the sense of Hyers}

A map $f: A \rightarrow F$ is said to be of type $\left(D^{-1}\right)$ at $a \in A$ in the sense of Hyers if it satisfies the following three conditions:

(1) $f$ is an injective map onto an open set $f(A)$; 
(2) $f$ is Hyers differentiable at $a \in A$ and $f^{\prime}(a)$ is an isomorphism;

(3) the inverse map $g$ of $f$ is $(\mathrm{H})$-continuous at $f(a)$.

As we shall see in the next section, the inverse map of a map $f: A \rightarrow F$ of type $\left(D^{-1}\right)$ at $a \in A$ in the sense of Hyers is not necessarily Hyers differentiable at $f(a)$. Therefore, we need to find a necessary and sufficient condition for the inverse map to be Hyers differentiable at $f(a)$. The situation is similar to the case of Fréchet differentiability treated in Yamamuro (to appear; 1975), and we introduce a notion which is similar to the braked continuity defined in Yamamuro (1975).

Let $\left(\alpha_{n}\right) \subset R$ be a sequence such that $\alpha_{n} \rightarrow+\infty$ and $p \in P(E)$. Then, a sequence $\left(x_{n}\right) \subset E$ is said to be $\left(\alpha_{n}, p\right)$-braked if $\left(p\left(\alpha_{n} x_{n}\right)\right)$ is a bounded sequence. A map $f: A \rightarrow F$ is said to be brakedly $(H)$-continuous at $a \in A$ if , for any $q \in P(F)$, there exists $p \in P(E)$ such that $\left(f\left(a+x_{n}\right)-f(a)\right)$ is $\left(\alpha_{n}, q\right)$ braked whenever $\left(x_{n}\right)$ is $\left(\alpha_{n}, p\right)$-braked.

(2.1) Let $f: A \rightarrow F$ be of type $\left(D^{-1}\right)$ at $a \in A$ in the sense of Hyers. Then, $f$ is brakedly $(H)$-continuous at a.

Proof. Let $q \in P(F)$; then there exists $p \in P(E)$ such that

$$
\lim _{\varepsilon \rightarrow 0} \sup _{p(x) \leq 1} q\left(\varepsilon^{-1}[f(a+\varepsilon x)-f(a)]-f^{\prime}(a)(x)\right)=0 .
$$

Let $\left(x_{n}\right)$ be an $\left(\alpha_{n}, p\right)$-braked sequence. We can assume that $p\left(\alpha_{n} x_{n}\right) \leqq 1$ for all $n, p \geqq q$ and $q\left(f^{\prime}(a)(x)\right) \leqq p(x)$ for all $x$. Then, since

$$
q\left(\alpha_{n}\left[f\left(a+x_{n}\right)-f(a)\right]-f^{\prime}(a)\left(\alpha_{n} x_{n}\right)\right) \rightarrow 0,
$$

we have

$$
\begin{aligned}
q\left(\alpha_{n}\left[f\left(a+x_{n}\right)-f(a)\right]\right) & \leqq 1+q\left(f^{\prime}(a)\left(\alpha_{n} x_{n}\right)\right. \\
& \leqq 1+p\left(\alpha_{n} x_{n}\right) \leqq 2,
\end{aligned}
$$

which implies that $\left(f\left(a+x_{n}\right)-f(a)\right)$ is a $\left(\alpha_{n}, q\right)$-braked sequence.

(2.2) Let $f: A \rightarrow F$ be of type $\left(D^{-1}\right)$ at $a \in A$ in the sense of Hyers. Then, the inverse map $g$ is Hyers differentiable at $f(a)$ if and only if $g$ is brakedly $(H)$-continuous at $f(a)$.

Proof. If $g$ is Hyers differentiable at $f(a)$, then $g$ is of type $\left(D^{-1}\right)$ at $f(a)$ in the sense of Hyers. Hence, by (2.1), it is brakedly (H)-continuous at $f(a)$.

Conversely, let $f$ be of type $\left(D^{-1}\right)$ at $a$ in the sense of Hyers. Then, the map

$$
x \rightarrow f^{\prime}(a)^{-1}[f(a+x)-f(a)]
$$

is of type $\left(D^{-1}\right)$ at zero in the sense of Hyers. Hence, we can assume that $f(0)=0, f$ is of type $\left(D^{-1}\right)$ at zero in the sense of Hyers and $f^{\prime}(0)=1$ (the identity map). 
Assume that $g$ is not Hyers differentiable at zero. Then, by (1.3), there exists $q \in P(E)$ such that

$$
\sup _{p(y) \leq 1} q\left(\varepsilon^{-1} g(\varepsilon y)-y\right) \not 00
$$

for any $p \in P(E)$. Since $f$ is Hyers differentiable and brakedly (H)-continuous at zero, there exist $p_{i} \in P(E)(i=1,2)$ such that

$$
\sup _{p_{1}(x) \leqq 1} q\left(\varepsilon^{-1} f(\varepsilon x)-x\right) \rightarrow 0
$$

and, for any sequence $\left(\alpha_{n}\right) \subset R$, every $\left(\alpha_{n}, p_{2}\right)$-braked sequence is $\left(\alpha_{n}, p_{1}\right)$ braked. Then, for this $p_{2}$, there exist $\left(\varepsilon_{n}\right) \subset R$ and $\left(y_{n}\right) \subset E$ such that $\varepsilon_{n} \rightarrow 0, p_{2}\left(y_{n}\right) \leqq 1$ and

$$
q\left({ }_{n}^{e-1} g\left(\varepsilon_{n} y_{n}\right)-y_{n}\right) \nrightarrow 0 .
$$

Put $x_{n}=\varepsilon_{n}^{-1} g\left(\varepsilon_{n} y_{n}\right)$. Since $\left(\varepsilon_{n} y_{n}\right)$ is an $\left(\varepsilon_{n}^{-1}, p_{2}\right)$-braked sequence, $\left(\varepsilon_{n} x_{n}\right)$ is $\left(\varepsilon_{n}^{-1}, p_{1}\right)$-braked, which means that $p_{1}\left(x_{n}\right) \leqq \gamma$ for some $\gamma$ and all $n$. Therefore,

$$
q\left(\varepsilon_{n}^{-1} g\left(\varepsilon_{n} y_{n}\right)-y_{n}\right)=q\left(x_{n}-\varepsilon_{n}^{-1} f\left(\varepsilon_{n} x_{n}\right)\right) \rightarrow 0,
$$

which is a contradiction.

\section{Spaces of type $\left(D^{-1}\right)$ in the sense of Hyers}

A space $E$ is said to be of type $\left(D^{-1}\right)$ in the sense of Hyers if, for any $F$ and any map $f: A \rightarrow F$ which is of type $\left(D^{-1}\right)$ at $a \in A$, the inverse map $g$ is Hyers differentiable at $f(a)$. The results in this section imply that every (F)-space which is of $\left(D^{-1}\right)$ in the sense of Hyers is normable.

A space $E$ is said to be $(H)$ levered if, for any null sequence $\left(x_{n}\right) \subset E$ such that $x_{n} \neq 0$ for all $n$, there exists $q \in P(E)$ such that the following condition is satisfied: for any $p \in P(E)$ there exists $\left(\alpha_{n}\right) \subset R$ such that $p\left(\alpha_{n} x_{n}\right) \leqq 1$ but $q\left(\alpha_{n} x_{n}\right) \nrightarrow 0$. Roughly speaking, the condition is equivalent to say that $q\left(x_{n}\right) / p\left(x_{n}\right) \nrightarrow 0$ for every $p \in P(E)$.

In Yamamuro (1974; page 148), we have called a space $E$ levered if, for any null sequence $\left(x_{n}\right) \subset E$ such that $x_{n} \neq 0$, there exists $\left(\alpha_{n}\right) \subset R$ such that $\left(\alpha_{n} x_{n}\right)$ does not converge to zero. It is evident that $(H)$-levered spaces are levered.

(3.1) If $E$ is of type $\left(D^{-1}\right)$ in the sense of Hyers, then it is $(H)$-levered.

Proof. Let $E$ be of type $\left(D^{-1}\right)$ in the sense of Hyers. First, let us assume that $E$ is not levered. Then, by Proposition (2.3) in Yamamuro (1974; page 148), there exists a null sequence $\left(y_{n}\right)$ such that $\alpha_{n} y_{n} \rightarrow 0$ for any $\left(\alpha_{n}\right) \subset R$ and there exists $\left(\bar{y}_{n}\right) \in \bar{E}$ (the dual of $E$ ) such that $\left(y_{n}, \bar{y}_{n}\right)$ is biorthogonal. 
We define a null sequence $\left(x_{n}\right)$ by

$$
x_{n}=2^{-2^{n}} y_{1}+2^{-2^{n-1}} y_{2}+\cdots+2^{-2} y_{n},
$$

and prove that for any $q \in P(E)$ there exists $p \in P(E)$ such that, if $p\left(\alpha_{n} x_{n}\right) \leqq 1$ for all $n$, then $q\left(\alpha_{n} x_{n}\right) \rightarrow 0$.

To prove this, let us take an arbitrary $q \in P(E)$. Then, it is easy to see that there exists $k$ such that $q\left(y_{n}\right)=0$ if $n \geqq k$. Then,

$$
p(x)=\sum_{i=1}^{k+1}\left|\left\langle x, \bar{y}_{i}\right\rangle\right|
$$

is a continuous semi-norm on $E$. Assume that $p\left(\alpha_{n} x_{n}\right) \leqq 1$ for all $n$. Then, if $n \geqq k$,

$$
q\left(\alpha_{n} x_{n}\right) \leqq \sum_{i=1}^{k} q\left(\alpha_{n} 2^{-2^{n-i+1}} y_{i}\right)
$$

Moreover, since

$$
\left|\alpha_{n} 2^{-2^{n} \cdot i+1}\right|=\left|\left\langle\alpha_{n} x_{n}, y_{i}\right\rangle\right| \leqq p\left(\alpha_{n} x_{n}\right) \leqq 1 \text { for } i=1,2, \cdots, k+1,
$$

we have

$$
\begin{aligned}
\left|\alpha_{n} 2^{-2^{n-1+2}}\right| & =\left|\alpha_{n} 2^{-2^{n-1+1}}\right|\left|2^{-2^{n-i+1}}\right| \\
& \leqq\left|2^{-2^{n-t+1}}\right| \rightarrow 0 \text { if } n \rightarrow \infty \text { and } 1 \leqq i \leqq k+1 .
\end{aligned}
$$

Hence, $q\left(\alpha_{n} x_{n}\right) \rightarrow 0$.

Now, from these two sequences $\left(x_{n}\right)$ and $\left(y_{n}\right)$, we construct the same map as we constructed in Proposition (2.4) of Yamamuro (1974; page 150). Namely, we first put

$$
x_{n, k}=\left(2-\frac{1}{k}\right) x_{n}, \quad y_{n, k}=\left(2-\frac{1}{k}\right) y_{n},
$$

and define a map $f: E \rightarrow E$ as follows:

$$
\begin{array}{ll}
f\left(x_{n, k}\right)=x_{n, k-1} & \text { for } n \geqq 1 \text { and } k \geqq 2, \\
f\left(x_{n}\right)=y_{n} & \text { for } n \geqq 1, \\
f\left(y_{n, k}\right)=y_{n, k+1} & \text { for } n \geqq 1 \text { and } k \geqq 1, \\
f(x)=x & \text { elsewhere. }
\end{array}
$$

Then, it is easy to see that $f$ is Hyers differentiable at zero and $f^{\prime}(0)=1$. Moreover, since $\left(x_{n}\right)$ and $\left(y_{n}\right)$ are null sequences, $g$ is $(H)$-continuous at zero.

Finally, we show that the inverse map $g: E \rightarrow E$ is not Hyers differentiable at zero, which means, by (1.3), that the identity map is not the Hyers 
derivative of $g$ at zero. To do so, we choose $q \in P(E)$ and $\left(\alpha_{n}\right) \subset R$ such that $\alpha_{n} \rightarrow \infty$ and $q\left(\alpha_{n} x_{n}\right) \nrightarrow 0$. For instance, $q(x)=\left|\left\langle x, y_{1}\right\rangle\right|$ will do. Put $z_{n}=\alpha_{n} y_{n}$. Then, since $z_{n} \rightarrow 0$, for any $p \in P(E), p\left(z_{n}\right) \leqq 1$ except for at most finite $n$. Moreover,

$$
q\left(\alpha_{n} g\left(\alpha_{n}^{-1} z_{n}\right)-z_{n}\right)=q\left(\alpha_{n} g\left(y_{n}\right)-\alpha_{n} y_{n}\right)=q\left(\alpha_{n} x_{n}-\alpha_{n} y_{n}\right) \not 0,
$$

which means that the identity map is not the Hyers derivative of $g$ at zero. Thus, it has been proved that a space of type $\left(D^{-1}\right)$ in the sense of Hyers is levered.

Now, assume that $E$ is not $(H)$-levered. Then, there exists a null sequence $\left(e_{n}\right) \subset E$ such that $e_{n} \neq 0$ for all $n$ with the following property: for any $q \in P(E)$ there exists $p \in P(E)$ such that $p\left(\alpha_{n} e_{n}\right) \leqq 1$ implies $q\left(\alpha_{n} e_{n}\right) \rightarrow 0$. Since $E$ is levered, there exists $\left(\rho_{n}\right) \subset R$ such that $\left(\rho_{n} e_{n}\right)$ is not null. Since every finite dimensional subspace is $(H)$-levered, we can assume that, by taking a subsequence if necessary, that $\left(e_{n}\right)$ is linearly independent and $\rho_{n} \rightarrow \infty$.

Next, we construct the same map as in Proposition (3.3) of Yamamuro (1974; page 153). Namely, we take $\left(\lambda_{n}\right) \subset R$ and $\left(\mu_{n}\right) \subset R$ such that $\lambda_{n} \rightarrow 0, \mu_{n} \rightarrow 0$ and $\mu_{n}^{-1} \lambda_{n}=\rho_{n}+1$, and put

$$
\lambda_{n, k}=\left(2-\frac{1}{k}\right) \lambda_{n} \text { and } \mu_{n, k}=\left(2-\frac{1}{k}\right)^{-1} \mu_{n} .
$$

Then, define a map $f: E \rightarrow E$ as follows:

$$
\begin{array}{ll}
f\left(\lambda_{n, k} e_{n}\right)=\lambda_{n, k-1} 3_{n} & \text { for } n \geqq 1 \text { and } k \geqq 2, \\
f\left(\lambda_{n} e_{n}\right)=\mu_{n} e_{n} & \text { for } n \geqq 1, \\
f\left(\mu_{n, k} e_{n}\right)=\mu_{n, k+1} e_{n} & \text { for } n \geqq 1 \text { and } k \geqq 1, \\
f(x)=x & \text { elsewhere. }
\end{array}
$$

Then, since $\left(e_{n}\right)$ is linearly independent, $f$ is an injective map of $E$ onto $E$. In order to prove that the identity map is the Hyers derivative of $f$ at zero, we take an arbitrary $q \in P(E)$. Then, by the assumption, there exists $p \in P(E)$ such that $p\left(\alpha_{n} e_{n}\right) \leqq 1$ implies $q\left(\alpha_{n} e_{n}\right) \rightarrow 0$. Hence, it is easy to see that

$$
\sup _{p(x) \leq 1} q\left(\varepsilon^{-1} f(\varepsilon x)-x\right) \rightarrow 0
$$

for these $p$ and $q$. It is also obvious that $g$ is $(H)$-continuous at zero, because $\left(\lambda_{n}\right),\left(\mu_{n}\right)$ and $\left(e_{n}\right)$ are null sequences.

If $g$ is Hyers differentiable at zero, then $g^{\prime}(0)=1$. However, this is impossible because, if we take $q \in P(E)$ such that $q\left(\rho_{n} e_{n}\right) \nrightarrow 0$, then

$$
q\left(\mu_{n}^{-1} g\left(\mu_{n} e_{n}\right)-e_{n}\right)=q\left(\mu_{n}^{-1} \lambda_{n} e_{n}-e_{n}\right)=q\left(\rho_{n} e_{n}\right) \not 0,
$$


and, moreover, for each $p \in P(E), p\left(e_{n}\right) \leqq 1$ except for finite $n$.

Thus, if $E$ is of type $\left(D^{-1}\right)$, then $E$ is $(H)$-levered.

It is easy to see that every normed space and every strict inductive limit of an increasing sequence of Banach spaces are $(H)$-levered. The next result shows that there are not many $(H)$-levered spaces besides these.

(3.2) If an (F)-space is (H)-levered, then it is normable.

Proof. If an $(F)$-space $E$ is not normable, then the topology is defined by an increasing sequence of semi-norms $\left(p_{i}\right)$ whose members are mutually non-equivalent. Hence, in the same manner as in Proposition (3.3) of Yamamuro (to appear (a)), we can choose a sequence $\left(x_{n}\right) \subset E$ such that

$$
p_{1}\left(x_{n}\right) \geqq 1 \text { and } n p_{i}\left(x_{n}\right)<p_{i+1}\left(x_{n}\right) \text { if } 1 \leqq i, n<\infty \text {. }
$$

Then, for $\alpha_{n}=1 / n p_{n}\left(x_{n}\right),\left(\alpha_{n} x_{n}\right)$ is a null sequence and $\alpha_{n} x_{n} \neq 0$ for all $n$. Then, for each $i$,

$$
p_{i}\left(\alpha_{n} x_{n}\right) / p_{i+1}\left(\alpha_{n} x_{n}\right)<\frac{1}{n} \rightarrow 0
$$

which means that $E$ is not $(H)$-levered.

\section{Remarks}

If $E$ and $F$ are normed spaces, $f: A \rightarrow F$ is Fréchet differentiable at $a \in A$ and $f^{\prime}(a)$ has a continuous inverse, then $f$ is weakly injective at $a$, that is, there exists a neighbourhood $U$ of zero such that $f(a) \neq f(a+x)$ whenever $x \in U$. In Yamamuro (to appear (a)), we have shown that this fact remains true for locally convex spaces under the strong Fréchet differentiability but it fails under the Fréchet differentiability. The map $f$ of a countable product of reals defined by

$$
f(x)=\left(\xi_{n}-\xi_{n}^{2}\right) \quad \text { for } \quad x=\left(\xi_{n}\right),
$$

which was first presented by Keller (1963-64), is Hyers differentiable at zero and $f^{\prime}(0)=1$, but it is not weakly injective at zero.

From these observations, it seems to be reasonable to conclude that there is not much sense in using the Hyers differentiability instead of more convenient Fréchet differentiability. The only significant difference between these two differentiabilities is that the former implies continuity whereas the latter does not. However, in view of the remark given after Proposition (1.2), we can not regard this as an advantage of the Hyers differentiability. In fact, a differentiability is useful if either it does not imply continuity and satisfies the chain rules of all orders (for example, Fréchet differentiability) or it behaves well in such problems as differentiability of the inverse maps and weak 
injectivity under natural conditions (for example, strong Fréchet differentiability). The Hyers differentiability does not have any of these properties.

In Yamamuro $(1974 ; 1.12)$, we have seen that, among all differentiabilities proposed so far, there is only one differentiability which is stronger than the Hyers differentiability; it is the strong Fréchet differentiability.

\section{References}

D. H. Hyers (1945), 'Linear topological spaces', Bull. Amer. Math. Soc. 51, 1-21.

H. H. Keller (1963-64), 'Differenzierbarkeit in topologischen Vektorräumen', Comm. Math. Helv., 38, 308-320.

S. Yamamuro (1974), Differential Calculus in Topological Linear Spaces, (Lecture Note in Math., Vol. 374, Springer-Verlag, 1974).

S. Yamamuro (to appear), 'Notes on differential calculus in topological linear spaces', J. Reine Angew Math.

S. Yamamuro (1975), 'Notes on differential calculus in topological linear spaces, II', J. Austral. Math. Soc. 20 (Series A), 245-252.

Department of Mathematics Institute of Advanced Studies Australian National University

Canberra, A.C.T. 